\title{
Caracterização fenotípica e genética da produção de leite e do intervalo entre partos em bubalinos da raça Murrah
}

\author{
Alcides de Amorim Ramos ${ }^{(1)}$, Carlos Henrique Mendes Malhado(2), Paulo Luiz Souza Carneiro(2), \\ Heraldo César Gonçalves ${ }^{(1)}$ e Danielle Maria Machado Ribeiro Azevedo(3)
}

\begin{abstract}
(1) Universidade Estadual Paulista, Fac. de Medicina Veterinária e Zootecnia, Dep. de Produção e Exploração Animal, Fazenda Lageado, Caixa Postal 560, CEP 18618-000 Botucatu, SP. E-mail: aaramos@fca.unesp.br (2)Universidade Estadual do Sudoeste da Bahia, Dep. de Ciências Biológicas, Av. José Moreira Sobrinho, s/no, Jequiezinho, CEP 45200-000 Jequié, BA. E-mail: carlosmalhado@gmail.com, plscarneiro@gmail.com ${ }^{(3)}$ Embrapa Meio-Norte, UEP/Parnaíba, BR 343, Km 35, Caixa Postal 341, CEP $64200-970$ Parnaíba, PI. E-mail: danizootec3@hotmail.com
\end{abstract}

Resumo - O objetivo deste trabalho foi estimar as correlações, herdabilidades, repetibilidades, tendências genéticas e fenotípicas, e avaliar as distribuições univariada e bivariada da produção de leite e do intervalo entre partos, em fêmeas bubalinas da raça Murrah, paridas no período de 1982 a 2003 . As tendências genéticas e fenotípicas foram estimadas pelas regressões das variáveis dependentes sobre o ano de parto, pelos métodos: regressão linear e regressão não paramétrica, utilizando-se a função de alisamento Spline. As herdabilidades estimadas foram 0,21 e 0,02, e as repetibilidades, 0,32 e 0,06, para a produção de leite e intervalo entre partos, respectivamente. As correlações genética, fenotípica e ambiental foram -0,22, 0,01 e 0,03, respectivamente. As tendências genéticas (regressão linear) foram significativas e iguais a 1,57 kg por ano e 0,085 dia por ano, e as tendências fenotípicas foram $27,74 \mathrm{~kg}$ por ano e 0,647 dia por ano, para a produção de leite e intervalo entre partos, respectivamente, tendo sido significativa apenas para a produção de leite. A correlação negativa sugere a existência de antagonismo favorável entre produção de leite e intervalo entre partos; assim é possível selecionar animais com altos valores genéticos para a produção de leite e com menores valores para o intervalo entre partos.

Termos para indexação: análise bivariada, correlações, herdabilidade, tendência genética.

\section{Phenotypic and genetic characterization of the milk yield and calving interval in buffalo of the Murrah breed}

\begin{abstract}
The objective of this work was to estimate the correlations, heritabilities, repeatabilities, phenotypic and genetic trends, and to evaluate the univariate and bivariate distributions of the milk yield and calving interval of Murrah breed buffalo, calved from 1982 to 2003. The genetic and phenotypic trends were estimated by regressions of the dependant variables on year of the animals calving, based on two methods: linear regression and articulated polynomial regression. The heritabilities estimates were 0.21 and 0.02 , and the repeatabilities 0.32 and 0.06, for milk yield and calving interval, respectively. The genetic, phenotypic and environmental correlations were $-0.22,0.01$ and 0.03 , respectively. Genetics trends (linear regression) were significant and equal to $1.57 \mathrm{~kg}$ per year and 0.085 day per year, and the phenotypics trends were $27.74 \mathrm{~kg}$ per year and 0.647 day per year, for milk yield and calving interval, respectively, being significant just for milk yield. The negative genetic correlation suggests that there is a favorable relationship between milk yield and calving intervals, thus it is possible to select animals of high breeding values for milk yield and with low breeding values for calving intervals.
\end{abstract}

Index terms: bivariate analysis, correlation, heritability, genetic trend.

\section{Introdução}

Há muitas décadas, os bubalinos vêm sendo criados no Brasil e, atualmente, existem cerca de 2,8 milhões distribuídos por todos os estados. Essa espécie tem apresentado grande expansão em nosso território, com crescimento de 10\% ao ano. Em diversos estados brasilei- ros, os bubalinos têm-se tornado uma boa opção econômica, principalmente, pela exploração leiteira e elaboração do queijo mozarela. Esse produto apresenta boa aceitação pelo mercado, e é comercializado com ótimo preço, decorrente da baixa oferta (Tonhati, 2002).

O retorno econômico da bubalinocultura de leite depende da produção de leite e da eficiência reprodutiva 
dos animais, esta última particularmente afetada pelo intervalo entre partos.

A produção de leite é tradicionalmente o principal objetivo de seleção em animais leiteiros. Entretanto, vários estudos têm mostrado declínio da eficiência reprodutiva, associada ao aumento da produção de leite (Silva et al., 1998).

Antagonismos genéticos que envolvem diferentes medidas de eficiência reprodutiva e produção de leite foram relatadas em bovinos por Bagnato \& Oltenaeu (1994), Marti \& Funk (1994) e Silva et al. (1998). Entretanto, pouco antagonismo entre eficiência reprodutiva e produção de leite foram observadas por Barnes et al. (1990). Zambianchi et al. (1999) relataram correlação genética positiva, porém, indesejável entre produção de leite e intervalo entre partos.

Abdallah \& McDaniel (2000) frisaram que nos vários trabalhos disponíveis na literatura com bovinos, em que foi avaliada a tendência genética, foram analisados basicamente os registros de produção de leite e de seus componentes, e não foram consideradas as possíveis alterações nas características reprodutivas, em decorrência da seleção direta, ou como resposta correlacionada da seleção para as características de produção.

Embora muitos trabalhos tenham sido realizados no país, com vistas a conhecer influências de ambiente e genética nas mais diferentes espécies comerciais, há deficiência de estimativas dos parâmetros genéticos e fenotípicos na bubalinocultura. Associado a isso, o conhecimento do progresso genético alcançado pelos diferentes rebanhos é desconhecido, tanto por criadores que realizam a seleção de maneira empírica, como por aqueles que participam de programas de melhoramento genético. Assim, torna-se necessário estudar a tendência genética ao longo do tempo não apenas com o objetivo de avaliar o progresso genético alcançado, mas também para nortear ações futuras.

O objetivo deste trabalho foi estimar as correlações, herdabilidades, repetibilidades, tendências genéticas e fenotípicas, e avaliar as distribuições univariada e bivariada da produção de leite e do intervalo entre partos, em fêmeas bubalinas da raça Murrah, paridas no período de 1982 a 2003.

\section{Material e Métodos}

A base de dados usada pertence ao programa de melhoramento genético de bubalinos (Ramos et al.,
2004), e neste estudo foram utilizadas informações de 3.392 fêmeas da raça Murrah, de 12 rebanhos, paridas no período de 1982 a 2003. A duração das lactações variou entre 150 e 390 dias, com média de 256 dias.

Para obter as estimativas de covariância e variância, foi realizada análise bivariada, empregando-se o método da máxima verossimilhança restrita livre de derivada (DFREML), por meio de modelo animal, utilizando-se o aplicativo Multiple Traits Derivate Free Restrict Maximum Likelihood (MTDFREML), desenvolvido por Boldman et al. (1995).

O modelo contém os efeitos aleatórios, aditivo direto e de ambiente permanente materno, e os efeitos fixos de época (duas estações e 21 anos de parto), fazenda (nove) e número de ordenhas (duas), além das covariáveis idade da matriz ao parto (linear e quadrática), para as duas características, e duração da lactação (linear), para a produção de leite.

As estimativas da tendência genética para a característica foram obtidas pela regressão dos valores genéticos sobre o ano de parto pelos métodos: regressão linear e regressão por polinômios articulados, utilizandose, o estimador Spline, que tem a forma:

$\mathrm{S}(\lambda)=\frac{1}{3} \sum_{\mathrm{i}=1}^{\mathrm{n}}\left(\mathrm{y}_{\mathrm{i}}-\hat{\mathrm{f}}_{\lambda}(\mathrm{x})\right)^{2}+\lambda \int_{-\infty}^{\infty}\left(\hat{\mathrm{f}}_{\lambda}(\mathrm{x})\right)^{2} \mathrm{dx}$

em que $\hat{\mathrm{f}}_{\lambda}$ pertence ao conjunto das funções continuadamente diferenciadas, com derivadas de segunda ordem de integráveis quadradas, onde $\lambda$ é uma constante positiva. O estimador resultante da minimização $S(\lambda)$ é chamado de estimador de alisamento Spline, que ajusta um polinômio cúbico em cada intervalo entre pontos. Para cada ponto $\mathrm{x}_{\mathrm{i}}$, a curva e suas primeiras duas derivadas são contínuas.

$O$ parâmetro $\lambda$ controla a intensidade de alisamento e a troca entre a bondade e o alisamento do ajuste. Seleciona-se um parâmetro de alisamento $\lambda$ por especificar uma constante c na fórmula: $\lambda-(\mathrm{Q} / 10) 3 \mathrm{c}$, em que $\mathrm{Q}$ é o intervalo interquartílico. Esta fórmula faz com que c seja independente da unidade de $\mathrm{x}$.

Para analisar as distribuições univariadas das características, foram empregadas duas densidades, com base na distribuição normal: a paramétrica, com a média e desvio-padrão obtidos da amostra, e a não-paramétrica, ou estimador Kernel, que tem a forma: $\mathrm{f} \lambda(\hat{\mathrm{y}})=1 /(\mathrm{n} \lambda)\left[\sum_{\mathrm{i}=1}^{\mathrm{n}} \mathrm{K}_{0}\left(\overline{\mathrm{x}}-\mathrm{x}_{\mathrm{i}}\right) / \lambda\right]$ em que $\mathrm{K}_{0}$ é a função Kernel, $\lambda$ é a largura da banda, ou 
seja, o parâmetro de alisamento dos dados, e $\mathrm{x}_{1}, \mathrm{x}_{2}, \ldots, \mathrm{x}_{\mathrm{n}}$ são variáveis aleatórias contínuas. A função de densidade normal padronizada, geralmente utilizada como função Kernel, é:

$\mathrm{K}_{0}(\mathrm{t})=1 /(2 \pi)^{0,5} \exp \left(-\mathrm{t}^{2} / 2\right)$, para $-\infty<\mathrm{t}<\infty$.

A discrepância entre o estimador, pela função Kernel, e a densidade verdadeira $\mathrm{f}(\mathrm{x})$ pode ser quantificada pela curva do quadrado médio do erro integrado aproximado (AMISE), dado por:

$\operatorname{AMISE}(\lambda)=(1 / 4) \lambda^{4}\left(\int_{t^{2}} \mathrm{~K}(\mathrm{t}) \mathrm{dt}\right)^{2} \int_{\mathrm{x}}\left(\mathrm{f}^{\mathrm{n}}(\mathrm{x})\right)^{2} \mathrm{dx}+$ $(1 / \mathrm{n} \lambda) \int_{\mathrm{t}} \mathrm{K}(\mathrm{t})^{2} \mathrm{dt}$

em que o parâmetro de alisamento $(\lambda)$ ótimo é aquele que minimiza AMISE.

Para análise da distribuição bivariada das características e de seus valores genéticos, utilizaram-se gráficos "scatter plot" e elipses de confiança para a predição com 95\% de probabilidade.

Uma elipse de confiança para predição é uma região de confiança para se predizer novas observações na população. Pode ser considerada como uma região com porcentagem específica da população.

Considerando-se Z como uma variável aleatória bivariada para uma nova observação e a variável $Z-\bar{Z}$ como uma variável bivariada normal - com média 0 , covariância 1 e independente de $\mathrm{S}$-, uma elipse de confiança de $100(1-\alpha) \%$ é dada pela equação: $(\mathrm{Z}-\overline{\mathrm{Z}})^{\prime} \mathrm{S}^{-1}(\mathrm{Z}-\overline{\mathrm{Z}})=\frac{2(\mathrm{n}+1)(\mathrm{n}-1)}{\mathrm{n}(\mathrm{n}-2)} \mathrm{F}_{2, \mathrm{n}-2(1-\alpha)}$

A correlação entre os pares de variáveis x e y é interpretada segundo a inclinação da elipse, ou seja, quando esta se assemelha a um círculo não há correlação e conforme o alongamento vai afunilando em direção a um ângulo de $45^{\circ}$ entre os eixos x e y, há correlação de moderada a alta. As análises foram realizadas com o SAS Institute, (1999).

\section{Resultados e Discussão}

As médias da produção de leite e do intervalo entre partos foram $1.650 \pm 659,5 \mathrm{~kg}$ e $432,4 \pm 123,4$ dias, e os coeficientes de variação, 39,9 e 28,5\%, respectivamente.

As estatísticas associadas às distribuições univariadas das duas características são apresentadas na Tabela 1. Na Figura 1, observa-se que a distribuição da produção de leite aproximou-se da normal. Entretanto, o intervalo entre partos apresentou uma distribuição leptocúrtica e assimétrica à esquerda, com acentuada discrepância em relação à distribuição normal.

Tabela 1. Estatística associada à função normal e Kernel para a produção de leite (PL) e intervalo entre partos (IEP), em bubalinos da raça Murrah(1).

\begin{tabular}{|c|c|c|c|c|c|c|c|c|c|}
\hline \multirow[t]{2}{*}{ Variável } & \multicolumn{5}{|c|}{ Função normal } & \multicolumn{4}{|c|}{ Função Kernel } \\
\hline & Média (kg) & $\mathrm{CV}(\%)$ & Moda (kg) & Curtose & Assimetria & $\lambda$ & $\mathrm{c}$ & Moda (kg) & AMISE \\
\hline $\begin{array}{l}\text { PL } \\
\text { IEP }\end{array}$ & $\begin{array}{r}1.650,0 \\
432,4\end{array}$ & $\begin{array}{l}39,9 \\
28,5\end{array}$ & $\begin{array}{r}1.650,0 \\
432,4\end{array}$ & $\begin{array}{l}1,01 \\
4,31\end{array}$ & $\begin{array}{l}0,94 \\
2,04\end{array}$ & $\begin{array}{r}130,67 \\
16,74\end{array}$ & $\begin{array}{l}0,7852 \\
0,7852\end{array}$ & $\begin{array}{r}1285,9 \\
361,5\end{array}$ & $\begin{array}{l}9,163 \mathrm{E}-07 \\
1,130 \mathrm{E}-05\end{array}$ \\
\hline
\end{tabular}

(1) $\lambda$ : parâmetro de alisamento; c: constante.
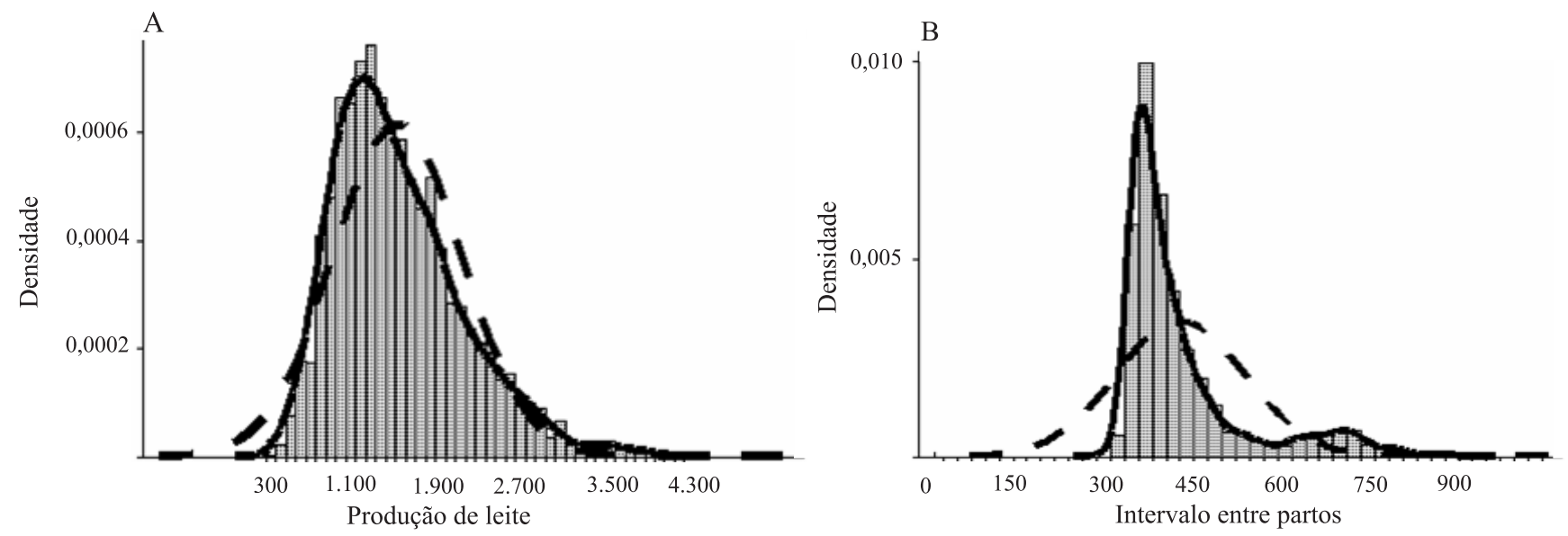

Figura 1. Estimativa da densidade paramétrica e Kernel para a produção de leite (A) e o intervalo entre partos (B). 
Em uma distribuição normal, a moda, a média e a mediana são iguais. As modas obtidas pelo estimador de Kernel para a produção de leite e intervalo entre partos foram 1.289,5 kg e 361,5 dias, respectivamente, e as determinadas pela função normal foram $1.650 \mathrm{~kg}$ e 432,4 dias. A divergência verificada na moda, obtida do ajuste de duas densidades, pode ser atribuída à existência de picos de freqüências associados à distribuição dos dados, os quais são responsáveis pelo desvio da distribuição em relação à normal paramétrica.

As estimativas das covariâncias, variâncias genética aditiva, de ambiente permanente, residual e fenotípica, bem como as herdabilidades e repetibilidades, para as duas características, estão descritas na Tabela 2.

A estimativa da herdabilidade para a produção de leite foi igual a 0,21 . Valores superiores foram observados por Kuralkar \& Raheja (1997) e Tonhati et al. (2000), que obtiveram herdabilidades de 0,25 e 0,24 , respectivamente. A magnitude dessas estimativas obtidas neste trabalho evidencia a existência de variabilidade genética aditiva, o que pode tornar eficiente a seleção para a característica estudada.

A herdabilidade para o intervalo entre partos foi de 0,02, valor em concordância com aqueles normalmente relatados na literatura sobre bovinos de leite, os quais são baixos (Abdallah \& McDaniel, 2000; Silva et al., 2001). Lôbo et al. (2000) revisaram informações de 52 artigos com bovinos leiteiros em regiões tropicais e relataram herdabilidade média de 0,11 para essa característica. Entretanto, estudos realizados com bubalinos por Penchev (1998), Mahdy et al. (1999), Aziz et al. (2001) e Cassiano et al. (2004) relatam valores iguais a 0,16, 0,17, 0,07 e 0,26, respectivamente.

As estimativas de herdabilidade do intervalo entre partos são obtidas com dados de campo, os quais estão sujeitos à interferência do criador; por exemplo, as me- didas de intervalo entre partos, geralmente, não incluem vacas descartadas por baixa produção ou por problemas reprodutivos, fato que proporciona uma diminuição da variância genética aditiva.

A repetibilidade estimada para a produção de leite foi 0,32 . Tonhati et al. (2000) estimaram repetibilidade de 0,38 para a produção de leite por lactação em búfalas. A magnitude da repetibilidade indica a possibilidade de se utilizar as primeiras produções das búfalas como indicativo de sua produção futura, e de escolher, assim, as fêmeas que continuarão no rebanho. A repetibilidade para o intervalo entre partos foi 0,06 , valor inferior ao relatado em búfalos $(0,14)$, por Aziz et al. (2001), e em bovinos leiteiros, por Abdallah \& McDaniel (2000), Pereira et al. (2000) e Silva et al. (2001).

A correlação genética entre as duas características foi de -0,22, o que evidencia a possibilidade de se obterem ganhos indiretos para reduzir o intervalo entre partos, por meio da seleção para produção de leite.

De acordo com Silva et al. (2001), pelo fato de a herdabilidade de intervalo entre partos ser próxima a zero, e de a correlação genética com a produção de leite ser moderada e negativa, pode ser interessante se proceder à seleção somente para o aumento da produção de leite. Entretanto, diversos trabalhos relatam que a correlação entre a produção de leite e o intervalo entre partos é positiva, como na revisão de Lôbo et al. (2000), que observaram correlação genética média de 0,08. Zambianchi et al. (1999) relataram correlação genética de 0,42 entre essas duas características e concluíram que a seleção para a produção de leite acarretaria aumento no intervalo entre partos.

As correlações fenotípica e ambiental foram iguais a 0,01 e 0,03, respectivamente. Este resultado indica que as características não são influenciadas pelos mesmos fatores ambientais e genéticos não aditivos, sendo

Tabela 2. Componentes de variância e covariância, herdabilidades e repetibilidades para a produção de leite (PL) e intervalo entre partos (IEP) em bubalinos da raça Murrah ${ }^{(1)}$.

\begin{tabular}{|c|c|c|c|c|c|c|c|c|}
\hline Variável & $\sigma^{2} \mathrm{a}$ & $\sigma^{2}$ pe & $\sigma^{2} \mathrm{e}$ & $\sigma^{2} p$ & $\sigma_{\mathrm{a} 1 \mathrm{a} 2}$ & $\sigma_{\text {pelpe2 }}$ & $\mathrm{h}^{2}$ & $r$ \\
\hline PL & $45.884,6$ & $25.551,6$ & $150.770,6$ & $199.206,8$ & $-772,9$ & $-814,9$ & 0,21 & 0,32 \\
\hline IEP & 222,6 & 529,08 & $13.746,0$ & $14.497,7$ & $-772,9$ & $-814,9$ & 0,02 & 0,06 \\
\hline
\end{tabular}

${ }^{(1)} \sigma^{2}$ a: variância genética aditiva; $\sigma^{2}$ pe: variância devido ao ambiente permanente; $\sigma^{2}$ e: variância ambiental; $\sigma^{2}$ p: variância fenotípica; $\sigma_{a 1 a 2}$ : covariância entre os efeitos aditivos diretos; $\sigma_{\mathrm{pe} 1 \mathrm{pe} 2}$ : covariância entre os efeitos de ambiente permanente; $\mathrm{h}^{2}$ : herdabilidade; r: repetibilidade. 
fenotipicamente independentes. A correlação fenotípica pode ser definida, estatisticamente, como a dependência entre as funções de distribuição de duas ou mais variáveis aleatórias. Assim, a discrepância da distribuição do intervalo entre partos justifica parte da baixa correlação fenotípica encontrada.

Os gráficos "scatter plot" corroboram os valores das correlações genética e fenotípica encontrados pela análise bivariada. Na Figura 2, nota-se ausência de correlação fenotípica, em razão de formato oval da elipse de confiança, entre as duas características. $\mathrm{O}$ formato mais alongado da elipse de confiança mostra fraca correlação negativa entre os valores genéticos das duas características (Figura 3). Essa elipse também pode ser denominada de região de tolerância para uma população, uma vez que na parte interna do círculo é esperado encontrar-se uma dada porcentagem da população, neste caso, $95 \%$.

Na Figura 4, observa-se regressão linear e não paramétrica para as duas características. As tendências genéticas (regressão linear) foram significativas $(\mathrm{p}<0,001)$ e iguais a $1,57 \mathrm{~kg}$ por ano $(\mathrm{y}=-3133,0+$ $1,573 x)$ e 0,086 dia por ano $(y=-171,877+0,086 x)$, para a produção de leite e intervalo entre partos, respectivamente. Em termos de mudanças genéticas anuais, isso representa 0,095 e 0,019\% das médias observadas para produção de leite e intervalo entre partos, respectivamente, que representam ganho genético de 33,03 kg para a produção de leite e redução de 1,80 dia para o intervalo entre partos, nos 21 anos estudados.

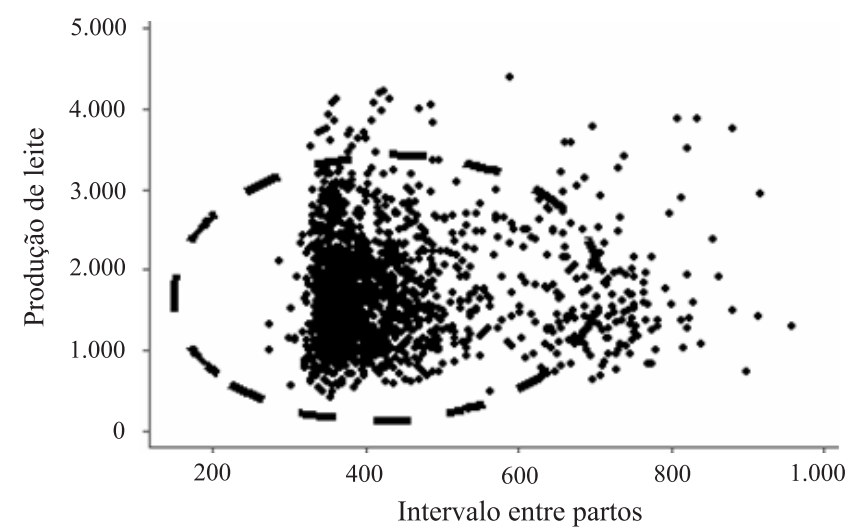

Figura 2. Gráfico “scatter plot” e elipses de confiança para a predição (95\%) da produção de leite e intervalo entre partos.
Considerando-se a intensidade de seleção de 1,305, isto é, retenção de $2 \%$ dos machos e $90 \%$ das fêmeas, herdabilidades de 0,21 e 0,02, desvios-padrão fenotípicos de 659,5 kg e 123,4 dias, e assumindo-se um intervalo entre gerações de cinco anos, seria possível obter ganhos genéticos $(\Delta G)$, por ano, da ordem de $36,1 \mathrm{~kg}$ e 3,2 dias, para a produção de leite e intervalo entre partos, respectivamente, na seleção direta para as duas características, desprezando-se a associação entre elas.

A resposta correlacionada para o intervalo entre partos, por meio da seleção apenas para a produção de leite, considerando-se as mesmas informações da seleção direta, seria de $-0,45$ dia por ano. Desta forma, a seleção apenas para a produção de leite traria resultados favoráveis no intervalo entre partos.

As tendências fenotípicas foram $27,74 \mathrm{~kg}$ por ano $(y=-53581,3+27,74 x)$ e 0,647 dias por ano $(y=-854,9$ $+0,646 x)$, para a produção de leite e intervalo entre partos, respectivamente, entretanto, foram significativas $(\mathrm{p}<0,001)$ apenas para a produção de leite.

Na Figura 5 A, observa-se alto ganho fenotípico entre 1988 e 1990. Este ganho é explicado pelo fato de que, a partir do ano de 1988, alguns produtores começaram a utilizar duas ordenhas diárias, ao invés de uma ordenha diária. Na Figura 5 B, observa-se, pela regressão não paramétrica, a oscilação entre os anos para o intervalo entre partos.

As tendências genéticas e fenotípicas observadas evidenciam que a maior parte do ganhos, no período,

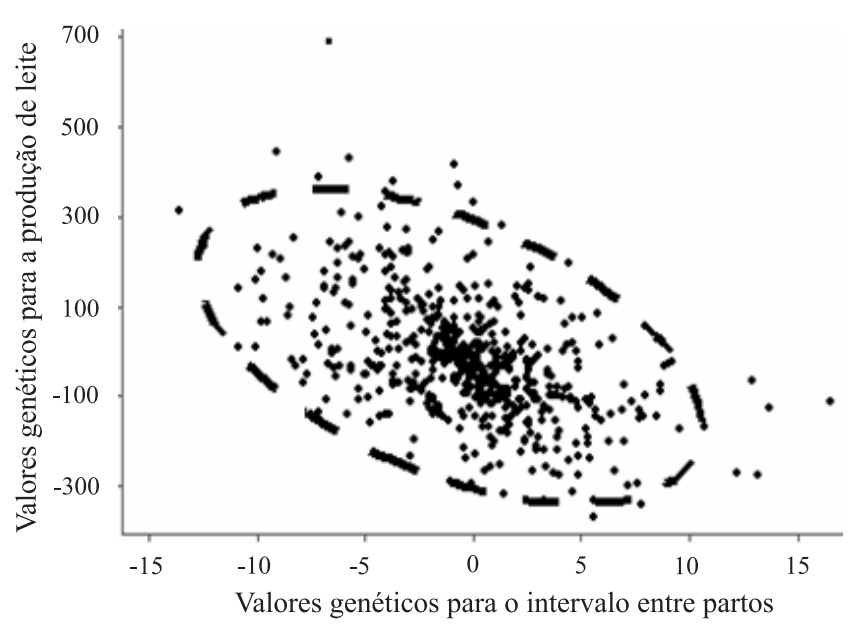

Figura 3. Gráfico "scatter plot” e elipses de confiança para a predição (95\%) dos valores genéticos da produção de leite e intervalo entre partos. 

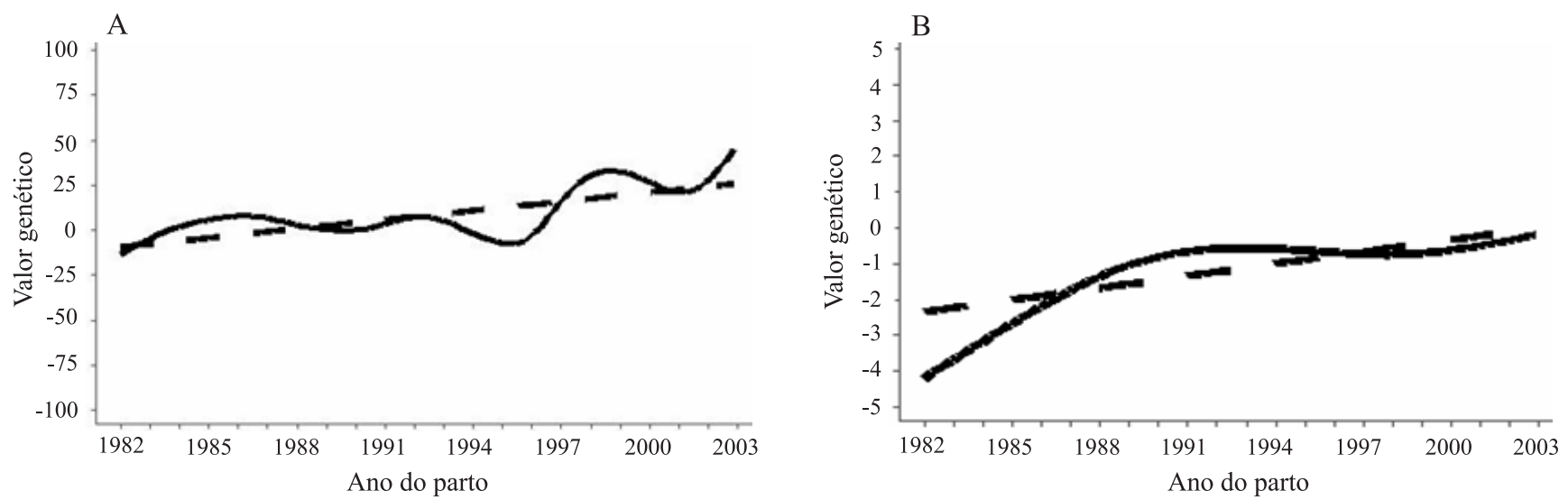

Figura 4. Tendência genética (regressão linear e não paramétrica) para a produção de leite (A) e o intervalo entre partos (B), em fêmeas bubalinas da raça Murrah.
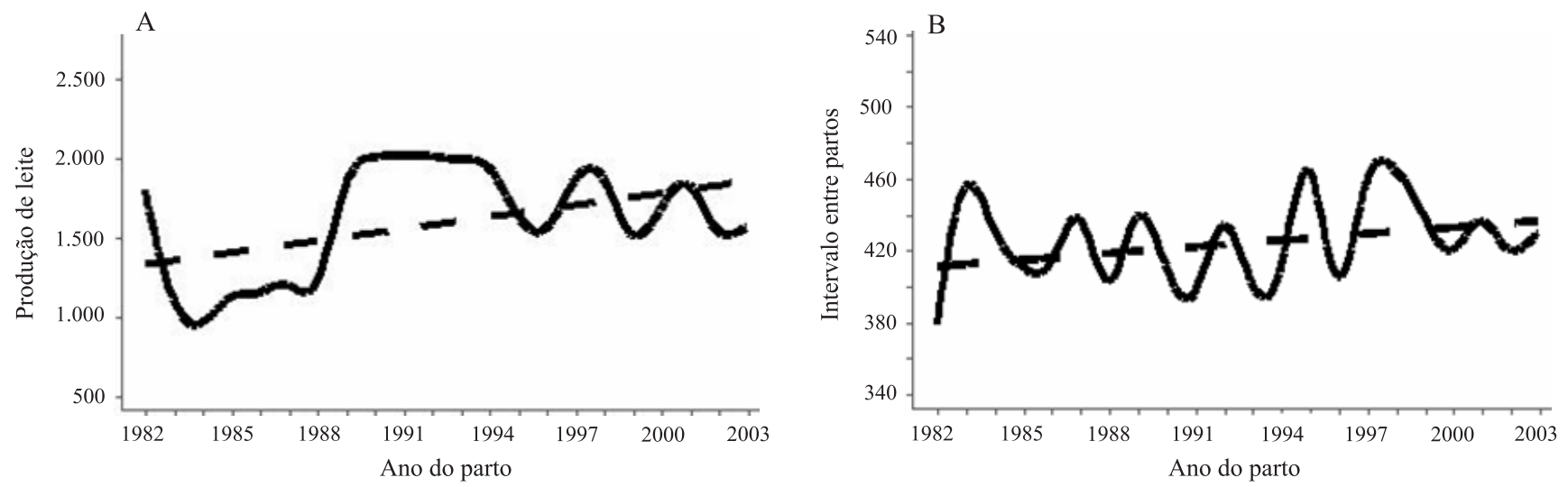

Figura 5. Tendência fenotípica (regressão linear e não paramétrica) para a produção de leite (A) e intervalo entre partos (B), em fêmeas bubalinas da raça Murrah.

ocorreu graças a melhorias nas condições ambientais, isto é, na alimentação/nutrição, sanidade, reprodução, manejo e instalações.

\section{Conclusões}

1. A magnitude da herdabilidade para a produção de leite é baixa, porém, passível de seleção, enquanto para o intervalo entre partos é quase nula.

2. A tendência genética para a produção de leite é positiva, porém aquém do desejado; a maior parte do ganho observado na característica ocorre em conseqüência do melhoramento das condições ambientais.

3. A correlação negativa sugere a existência de antagonismo favorável entre produção de leite e intervalo entre partos; desta forma, é possível selecionar animais com altos valores genéticos para a produção de leite e com valores negativos para o intervalo entre partos.

\section{Referências}

ABDALLAH, J.M.; McDANIEL, B.T. Genetic parameters and trends of milk, fat, days open and body weight after calving in North Carolina experimental herds. Journal of Dairy Science, v.83, p.1364-1370, 2000.

AZIZ, M.A.; SCHOEMAN, S.J.; JORDAAN, G.F.; EL-CHAFIE, O.M.; MAHDY, A.T. Genetic and phenotypic variation of some reproductive traits in Egyptian buffalo. South African Journal of Animal Science, v.31, p.195-199, 2001.

BAGNATO, A.; OLTENAEU, P.A. Phenotypic evaluation of fertility traits and their association with milk production of Italian Friesian cattle. Journal of Dairy Science, v.77, p.874-882, 1994. 
BARNES, M.A.; PEARSON, R.E.; LUKES-WILSON, A.J. Effects of milking frequency and selection for milk yield on productive efficiency of Holstein cows. Journal of Dairy Science, v.73, p.16031611, 1990.

BOLDMAN, K.G.; KRIESE, L.A.; VAN VLECK, L.D.; VAN JASSEL, C.P.V.; KACHMAN, S.D. A manual for use of MTDFREML: a set of programs to obtain estimates of variances and covariances [DRAFT]. Lincoln: Department of Agriculture, Agricultural Research Service, 1995. 120p.

CASSIANO, L.A.P.; MARIANTE, A.S.; McMANUS, C.; MARQUES, J.R.F.; COSTA, N.A. Parâmetros genéticos das características produtivas e reprodutivas de búfalos na Amazônia brasileira. Pesquisa Agropecuária Brasileira, v.39, p.451-457, 2004.

KURALKAR, S.V.; RAHEJA, K.L. Relationships among early performance, lifetime production and reproduction traits in Murrah buffaloes. Indian Journal of Animal Sciences, v.67, p.798-801, 1997.

LÔBO, R.N.B.; MADALENA, F.E.; VIEIRA, A.R. Average estimates of genetic parameters for beef and dairy cattle in tropical regions. Animal Breeding Abstracts, v.68, p.433-462, 2000.

MAHDY, A.E.; EL-SHAFIE, O.M.; AYYAT, M.S. Genetic study and sire values for some economic traits in Egyptian buffaloes. Alexandria Journal of Agricultural Research, v.44, p.15-35, 1999.

MARTI, C.F.; FUNK, D.A. Relationship between production and days open at different levels of herd production. Journal of Dairy Science, v.77, p.1682-1690, 1994.

PENCHEV, P. Phenotypic and genotypic evaluation of the buffalo population bred in Bulgaria. Bulgarian Journal of Agricultural Science, v.4, p.463-469, 1998.

PEREIRA, I.G.; GONÇALVES, T.M.; OLIVEIRA, A.I.G.; TEIXEIRA, N.M. Fatores de variação e parâmetros genéticos dos períodos de serviços e seco em bovinos da raça Holandês no Estado de Minas Gerais. Revista Brasileira de Zootecnia, v.29, p.10051013, 2000.

RAMOS, A. de A.; WECHSLER, F.S.; ONSELEN, V.J. van; GONCALVES, H.C. PROMEBUL: sumário de touros bubalinos. Botucatu: Unesp, 2004. 39p.

SAS INSTITUTE (Cary, Estados Unidos). User's guide: Stat. Cary, NC, 1999. 1v.

SILVA, M.V.G.B.; BERGMANN, J.A.G.; MARTINEZ, M.L.; PEREIRA, C.S.; FERRAZ, J.B.; SILVA, H.C.M. Associação genética, fenotípica e de ambiente entre medidas de eficiência reprodutiva e produção de leite na raça Holandesa. Revista Brasileira de Zootecnia, v.27, p.1115-1122, 1998.

SILVA, M.V.G.B.; COBUCI, J.A.; FERREIRA, W.J.; GUARAGNA, G.P.; OLIVEIRA, P.R.P. Respostas correlacionadas em características reprodutivas no programa de melhoramento do ecótipo Mantiqueira para produção de leite. Revista Brasileira de Zootecnia, v.30, p.1228-1235, 2001.

TONHATI, H. Critérios de seleção para produção total de leite em bubalinos criados no Estado de São Paulo, Brasil. 2002. 68p. Tese (Livre Docência) - Universidade Estadual Paulista, Jaboticabal.

TONHATI, H.; MUÑOZ, M.F.C.; OLIVEIRA, J.A.; DUARTE, J.M.C.; FURTADO, T.P.; TSEIMAZIDES, S.P. Parâmetros genéticos para produção de leite, gordura e proteína em bubalinos. Revista Brasileira de Zootecnia, v.29, p.2051-2056, 2000. Suplemento.

ZAMBIANCHI, A.R.; FREITAS, M.A.R.; PEREIRA, C.S. Efeitos genéticos e de ambiente sobre produção de leite e intervalos de partos em rebanhos leiteiros monitorados por sistemas de informação. Revista Brasileira de Zootecnia, v.28, p.1263-1267, 1999. 